\title{
Intrinsic Multiperspectivity: Conceptual Forms and the Functional Architecture of the Perceptual System
}

\author{
Rainer Mausfeld
}

\begin{abstract}
It is a characteristic feature of our mental make-up that the same perceptual input situation can simultaneously elicit conflicting mental perspectives. This ability pervades our perceptual and cognitive domains. Striking examples are the dual character of pictures in picture perception, pretend play, or the ability to employ metaphors and allegories. I argue that traditional approaches, beyond being inadequate on principle grounds, are theoretically ill equipped to deal with these achievements. I then outline a theoretical perspective that has emerged from a theoretical convergence of perceptual psychology, ethology, linguistics, and developmental research. On the basis of this framework, I argue that corresponding achievements are brought forth by a specific type of functional architecture whose core features are as follows: (1) a perceptual system that is biologically furnished with a rich system of conceptual forms, (2) a triggering relation between the sensory input and conceptual forms by which the same sensory input can be exploited by different types or systems of conceptual forms, and (3) computational principles for handling semantically underspecified conceptual forms. Characteristic features of the proposed theoretical framework are pointed out using the Heider-Simmel phenomenon as an example.
\end{abstract}

\section{Multiperspectivity}

The phenomenon in question can be vivaciously illustrated by a self-portrait of the French painter and caricaturist Alfred Le Petit, as depicted in Fig. 1. This painting visualises multiperspectivity in the literal sense of providing, within a single integral situation, multiple views of the same object as seen from different vantage points. At the same time, it allegorically illustrates a much more abstract feature

This chapter draws on material published in Mausfeld (2010a, b).

R. Mausfeld

Department of Psychology, Christian Albrechts University Kiel, Olshausenstr. 62, 24098 Kiel, Germany

e-mail: mausfeld@psychologie.uni-kiel.de 
of our mental make-up, namely our mental capacity to employ different "mental perspectives" in a given situation of sensory stimulation. For instance, if we mentally focus on the concrete painting hanging on a wall (or being reproduced in a book), the painted canvass is the salient object of perception. If, in another mental perspective, we focus on the depicted scene, we are mentally reading through the canvass, as it were, and regard it simply as a medium by which the depicted scene is conveyed to us as the salient object of perception. While the second mental perspective usually imposes itself more strongly than the first one, we have no problems adopting each of them and switching between the two. Each mental perspective constitutes a specific way of organising the sensory input in terms of meaningful categories and generates its own mental objects with their proprietary attributes. Hence, the assignments of attributes, such as colour, material qualities, depth or the degree of "realness", and their values, are subordinated to the organisation of the input in terms of perceptual objects. Each mental perspective is associated with and governed by its own specific forms of internal causal analyses. In the first type of mental perspective, these internal causal analyses notably pertain to the kind of operations to which a perceptual object of the type "canvass" is amendable. Accordingly, the four heads seen in Fig. 1 do not differ as to their type of "realness", namely being painted flat figures on a canvass. In the second mental perspective, these internal causal analyses pertain to a great variety of operations that are associated with the organisation of objects in three-dimensional perceptual space. Accordingly, each of the four portrayed heads in Fig. 1 is being associated with its own type and degree of "realness".

The painting shown in Fig. 1 exemplifies that the same situation of sensory stimulation can elicit in us quite different and even conflicting mental perspectives on what it is that we perceive. This capacity to employ different mental perspectives on what the senses offer to us is so blatantly obvious that we simply take it for granted

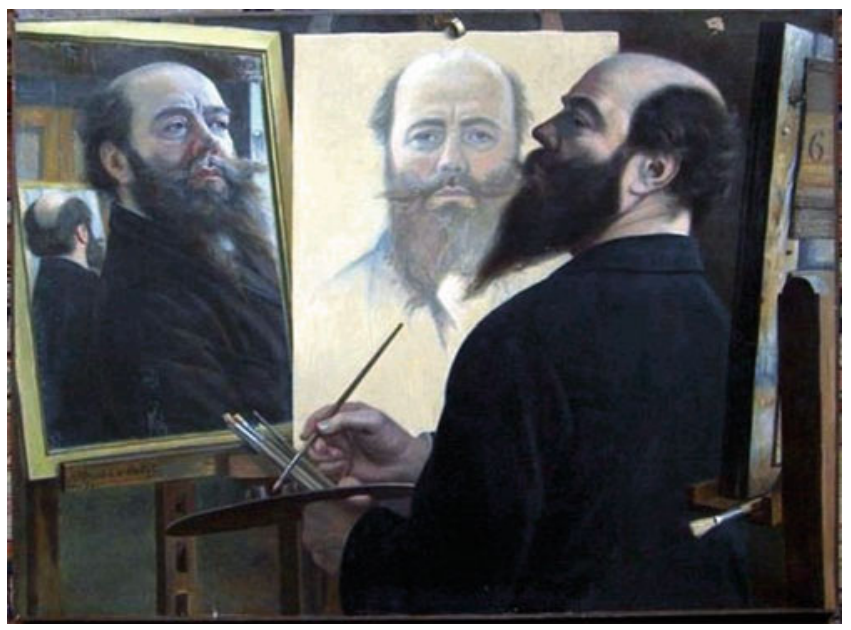

Fig. 1 Alfred Le Petit, Autoportrait, 1893 
in everyday life. It is an all pervading property of the way we are designed, and we are perpetually and effortlessly engaged in corresponding mental activities. As it is so pervading, it is, in the context of scientific enquiry, extraordinarily difficult for us to direct our attention to it and to find out on which specific features of our mental makeup it is based. However, once we begin scrutinising it, we will quickly become aware that the human capacity to engender, in a given situation, different mental perspectives is a uniquely powerful feature of our mind whose consequences for our perceptual and cognitive achievements one can hardly overestimate. Most importantly, this capacity provides a pre-eminent pillar for our competence to generate culture.

Although this capacity permeates all of our mental activity and thus remains largely inconspicuous, there are some situations that more easily draw our attention to it. For instance, in theatre or cinema, we perceive what happens on the stage or on the screen as segments of events going on in the world while at the same time being aware of actually watching a drama or a movie. With respect to a broad range of aspects, we can employ a mental double book-keeping, as it were, and simultaneously have different spatial, temporal, emotional, and other types of perspectives at our disposal. In the "duplication of space and time that occurs in theatrical representation", Michotte (1960/1991, p 191f.) noted, "the space of the scene seems to be the space in which the represented events are actually taking, or have taken, place and yet it is also continuous with the space of the theatre itself. Similarly for time also, instants, intervals, and successions for the spectators belong primarily to the events they are watching, but they are left nevertheless in their own present". The ability to perform this mental double book-keeping emerges quite early in our cognitive development. It notably manifests itself in the pretend play of children (Leslie 1987; cf. also Wyman and Racozcy, this volume), for which there is experimental evidence for children as young as 1-2 years old. Theatre and pretend play provide striking phenomena in which the availability of simultaneous mental perspectives almost becomes conspicuous at the surface of our phenomenal experience. They prototypically exemplify what William James has recognised as the hallmark of our mental life, namely that our "mind is at every stage a theatre of simultaneous possibilities" (James 1890/1983, p 277). Pretend play and other more conspicuous cases of a mental double book-keeping have prompted the question whether they can be explained by a common type of architectural principles of our mind. At the same time these striking phenomena have often impeded the recognition of the extent to which the capacity for simultaneous mental perspectives pervades all our perceptual achievements. The effects of this capacity are much harder to notice in apparently much more elementary situations. We can, for instance, perceive railroad tracks that recede from the observer towards the horizon both as being parallel and, in another mental perspective, as converging. We can perceive four points that are geometrically arranged as forming a square both as a concrete instance of a square and, in another mental perspective, as being four isolated points. We can perceive a circle that is partly occluded by another circle both as two integral circles lying at different depth levels, where the nearer one occludes part of the farther one, and, 
in another mental perspective, as two differently shaped objects lying side by side in a plane. We can perceive some location on a white wall at which a reddish spot light is directed as both appearing white, or, in another mental perspective, as appearing reddish. Traditionally, such phenomena have been treated under headings such as "proximal mode", "amodal completion", or "constancy phenomena". They have usually been assumed to predominantly result from highly special and often modality-specific mechanisms rather than from a common core of more abstract and general principles of our perceptual architecture. It is far from obvious, and, needless to say, an empirical question whether these different classes of phenomena express, to some theoretically interesting extent, a common abstract property of our mental machinery. However, the conjecture that they do seems to me warranted, on both theoretical and empirical grounds. These and a range of other phenomena share, or so I believe, at their core some structural aspects that point to much deeper principles of the conceptual and computational organisation of our perceptual system; more specifically to structural and dynamic aspects of the conceptual forms with which our perceptual system is biologically endowed and which provide the given "data format" for its internal computations. Before I venture, in the last section of this paper, some corresponding conjectures, I will try, in the Sects. 2 and 3, to identify some of the obstacles and misconceptions that have impeded an appropriate theoretical treatment of achievements expressing forms of multiperspecticity. In Sect. 4, I outline a general theoretical framework that appears to me promising, theoretically and empirically, for dealing with perception in general, and with certain phenomena of multiperspectivity that are grounded on specific design properties of the perceptual system, in particular. To bring out these cases, I introduce the notion of "intrinsic multiperspectivity". In Sect. 5, I will state more precisely and exemplify what I mean by "intrinsic multiperspectivity", and then venture, in Sect. 6, a theoretical conjecture as to its architectural and computational foundation.

Phenomena and perceptual achievements such as the ones mentioned before raise theoretical problems that are overwhelmingly complex and deep. Therefore, it is hardly surprising that we still lack agreement on even the most basic issues. Opinions are already deeply divided on what can be considered core phenomena that can be assumed to serve as a promising empirical basis for the sharp idealisations on which any successful explanatory account of perception has to be based. As analyses proceed, agreement about how to address the corresponding phenomena in theoretical terms will become even less likely, given the fact that our theoretical understanding of apparently much simpler achievements, such as the constitution of "perceptual objects", is rather thin (actually, this fundamental issue is almost entirely bypassed in prevailing approaches). Nevertheless, phenomena pertaining to simultaneous conflicting mental perspectives in perception cannot be regarded simply as side issues. Any theory of perception that neglects phenomena of multiperspectivity would be of marginal theoretical interest only. It poses, however, a particular challenge for perception theory that the variety of achievements that in our ordinary experience and in our ordinary discourse testify to some form of multiperspectivity can hardly be expected to rest on a single coherent set of 
principles. Rather such phenomena most likely mirror a variety of different aspects of the functional and computational architecture of our mind and involve complex interactions of various subsystems, such as the perceptual system proper, systems for imagination, higher-order interpretative systems, and language. The question then arises whether there are subclasses of such phenomena that can be assumed, on an appropriate level of idealisation, to result from specific internal properties and design principles of the perceptual system proper, understood as that modular component of our mental architecture on which our perceptual achievements are based. On the basis of an appropriate conception of the functional architecture of our perceptual system, a core of common principles becomes recognisable that yield phenomena of multiperspectivity as an essential consequence. In a later section, I will outline a corresponding theoretical framework that appears to me warranted on theoretical and empirical grounds and deal with ensuing principles that conjecturally generate phenomena of multiperspectivity. However, beforehand, I will turn the spotlight on the main obstacles that have fettered the development of an appropriate theoretical account of perception in general and have disposed perceptual psychology to pay little heed to phenomena of multiperspectivity.

\section{Common-Sense Intuitions on Perception}

Our common-sense conceptions ${ }^{1}$ of perception can be characterised as a blending of three different but related premises. The first premise pertains to what is regarded as the core achievement of perception, namely conveying a "truthful" representation of the external world. The second premise is intimately related to the first one and pertains to the properties and attributes of perception, which, according to our ordinary intuitions, are regarded to result from adaptations to our environment. The third premise pertains to the unit of analysis in terms of which we conceptualise perception in ordinary discourse, namely in terms of the unit of a person. ${ }^{2}$ I will briefly deal with these three aspects in turn.

\footnotetext{
${ }^{1}$ In speaking of common-sense conception about perception, I, in the present context, understand the term in the broadest possible way, namely as the diversity modes in which we conceive of perceptual phenomena and the process of perception itself in all contexts other than that of the natural sciences. This usage comprises not only those concepts and ways of world-making, which underlie, as part of our biological endowment, our ordinary discourse about the world and our acts of perceiving - sometimes referred to as "folk physics" and "folk psychology", but also derived concepts and notions pertaining to perceptual issues that have been developed for other purposes than those of the natural sciences, whether technological, philosophical, or of any other kind.

${ }^{2}$ Common-sense-based intuitions about perception are furthermore marked by a preference for "concretistic" conceptions of explanation in terms of tangible objects and concrete mechanism hence their fascination with neuroreductionist "explanations" and their dislike of more abstract accounts (cf. Mausfeld 2010c).
} 


\subsection{Perception and "Reality"}

According to our ordinary conception of perception, the senses convey to us an appropriate picture of the external world. Figure 2 illustrates, by the example of visual perception, how we basically conceive of the process of perception in our ordinary intuitions. At the core of our ordinary conception of perception thus is some kind of a (culturally refined) naïve realism, that is, the idea that the world, as it really and independently of an observer is, is mirrored in perception. We are convinced that perception basically works the way it phenomenally appears to us and that - apart from a few "perceptual illusions" - we perceive the world as it really is. Hence, we are strongly inclined to take the categories of our perceptual experiences as categories of the external world. ${ }^{3}$ On this account, perception is regarded as an entirely conspicuous process. At the same time, we are, of course, willing to accept some refinements and subtleties that are compelled by physics, which in turn claims to describe the world as it really is. This, however, does not diminish our conviction that our perception of the world is - apart from some subjective qualities and a few illusions - veridical.

At the core of our common-sense conception of perception is what is often been referred to as naïve realism, i.e. the idea that the world, as it really and independently of an observer is, is mirrored in perception. While naïve realism founders already in the face of the most elementary scientific facts say about the properties of our sense

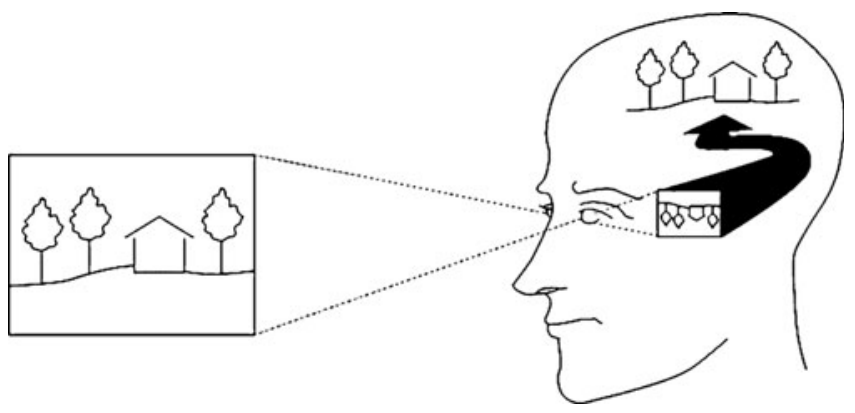

Fig. 2 The pictorial or similarity conception of perception

\footnotetext{
${ }^{3}$ The predisposition to take, in Kant's words, mere ideas for things in themselves, is the distinguishing mark of all of our mental activity. Kant thought of it as a "transcendental illusion". The transcendental illusion is the propensity to "take a subjective necessity of a connection of our concepts...for an objective necessity in the determination of things in themselves" (Critique of pure reason, A297/B354). Due to this propensity, the influence of which cannot be remedied by intellectual insight into it, we inevitably tend to mistake our own mental categories to hold "objectively" (cf. Grier 2001).
} 
organs, ${ }^{4}$ it captures some of our basic intuitions of the mental activity of perceiving, namely being in direct touch with a mind-independent world. The inappropriateness of our ordinary conception of perception becomes already obvious when we compare the world as it is represented in perception with the world as physics describes it. ${ }^{5}$ The kind of entities that populate our perceptual world are not the kind of abstract entities that populate the explanatory accounts of physics. There are only two ways of characterising what we suppose to be objects in the world, and thus of assigning a meaning to the attribute "real": One way is marked out by theoretical physics, which considers all these entities as objects of the world, and thus as real, that figure in its currently best explanatory accounts. The second way is delineated by our perceptual capacities and considers as objects of the world, and thus as "real", all the entities that can show up in our phenomenal experience. These ways are actually, as a result of our cultural development, inextricably interwoven in our scientifically and culturally moulded common sense. In the context of perception theory, it is crucial to be aware that we do not have (aside from theoretical physics) a way for characterising the "objects in the world" that is independent from our perceptual capacities. Thus, we cannot refer to objects in the world in a way that does not crucially depend on the output of the perceptual system. What we regard as "reality" accordingly is a product of or perceptual system. This already indicates that our concept of "reality" is the product of the specific perceptual and mental apparatus with which we are equipped. The fact that we have to presume sufficiently strong structural relations between the kind of reality as depicted by physics and the kind of reality as apparent in perception must not seduce us to mistake the meaningful categories of our mind with the way in which we describe the external world as independent of an observer. ${ }^{6}$

\footnotetext{
${ }^{4}$ Most significantly, the overwhelming part of the physical energy pattern that impinges on the organism is not perceived or used for biological purposes; we can, for instance, neither perceive the plane of polarisation level nor the direction of magnetic fields.

${ }^{5}$ Note, that even in cases, in which our linguistic vocabulary seems to suggest that our perceptual categories have a direct counterpart in the physical world, such as in the case of "illumination" or "surface", a closer examination reveals that physically defined categories and perceptually defined categories do not coincide and that, with respect to the functioning of the perceptual system, the occurrence of the former is neither necessary nor sufficient for the occurrence of the latter. The way we perceptually segment our world does not conform to the way we divide up things in the world when we are doing physics, an observation, which Ludlow (2003, p 150) terms a "type mismatch". ${ }^{6}$ Historically, our inclination to pursue our theoretical enquiries in such a way that they comply with common-sense intuitions is revealingly exemplified by the propagation of the notion of representation in perception theory. The widespread acceptance of this term, which has yielded tremendous philosophical confusion, historically seems to have been enhanced by the French translations of Descartes' profound and deep analyses of perception. Descartes used, e.g. in his Third Meditations, the Latin verbs "exhibeo" and "repraesento" interchangeably, in the sense of something that is internally presented to the mind. In seventeenth-century French both Latin verbs were translated by "representer", so that the meaning of the term shifted, due to the impact of common-sense intuitions, from denoting a predominantly mind-internal presentation to denoting a mind-world relation. While Descartes had an internalist meaning in mind, the French translation tacitly was guided by our ordinary preference for an externalist interpretation of the term.
} 


\subsection{Can the Structure of Perception be Derived from Adaptational Needs?}

Our conviction that the goal of senses is to convey to us a basically "truthful" account of the external world predisposes us to regard the specific properties and features of perception as predominantly formed by our biological adaptive needs. In perceptual psychology, corresponding intuitions find their expression in adaptational accounts of perception according to which the core properties of perception result from the biological adaptive needs to appropriately couple the organism to the external world. Because such adaptational intuitions derive from our commonsense conceptions, they unsurprisingly have a long history in perceptual psychology and still dominate, at least implicitly, the field. Spencer (1855, p 583) already presumed "that there exist in the nervous system certain pre-established relations answering to relations in the environment". He postulated a "continuous adjustment of internal relations to external relations". The structure of the mind is, according to Spencer, the "result from experiences continued for numberless generations", whereby the "uniform and frequent of these experiences have been successively bequeathed" in the process of evolution. James (1890, p 1222) lauded this as a "brilliant and seductive statement" that "doubtless includes a good deal of truth". It founders, however, according to James, "when the details are scrutinised, many of them will be seen to be inexplicable in this simple way". It is a matter of course that perception must structurally mirror or at least not contradict biological relevant aspects of the external world. This, however, is hardly an insight but rather simply rephrases from a functional point of view the kind of mental phenomena that have been singled out as an object of enquiry. From it, not much follows about the specific properties of the perceptual system. Even if the output of the perceptual system would not even in a single case mirror the true manner of being of the external world (however we might conceive of it), the perceptual system still could ensure a coupling of the organism to biologically relevant structural aspects of external word. ${ }^{7}$

To be sure, the perceptual system, as other computational or non-computational biological systems, has, in its evolutionary development, taken advantage of external physical regularities. From this, however, it neither follows that its core principles and its conceptual structure can be understood from these external regularities nor that considerations about adaptive purposes or about the "proper" external objects of perception play an explanatory role in accounts of the internal principles by which the perceptual system generates its outputs on the basis of specific inputs. ${ }^{8}$

\footnotetext{
${ }^{7}$ This insight also finds its expression in Helmholtz' sign theory (and in previous sign conceptions, notably Descartes').

${ }^{8}$ As in the case of other biological systems, an understanding of the internal functioning of the perceptual system does neither rest on a diachronic analysis of its selectional history. Explanatory accounts of perception furthermore do not rest on considerations of which physical entities should
} 
Furthermore, what we regard as a relevant regularity already depends on the specific nature of the system whose properties we intend to explain from those regularities. We can formulate literally infinitely many physical regularities, i.e. relations on sets of physico-mathematical entities that remain invariant under certain sets of transformations, of any degree of "unnaturalness", under which the perceptual system has evolved. There is no apriori notion of organism-relevant physical regularities. The nature of the organism determines which regions of the parameter space of the physical world are regarded as an environment. What constitutes a regularity depends on the structure of the organism under scrutiny, such as its size, the spatial and temporal integration properties of receptors and other neural structures, the properties of its memory, and its conceptual capacities.

Nevertheless, the idea that the nature of our perceptual system is formed by a "continuous adjustment of internal relations to external relations" is mostly regarded not as a hypothesis about the relation between specific external regularities and internal properties of the perceptual system but as a kind of self-evident truth. It then expresses the conviction that the fundamental properties of the perceptual system can be adequately accounted for by adaptational factors and that no essential explanatory importance needs to be attached to factors such as internal physical, architectural, or computational constraints in the evolutionary development of our mental architecture. Such a conviction again derives its apparent plausibility from common-sense intuitions. It sharply conflicts with the wealth of empirical evidence that has been gathered in Gestalt psychology and other areas of cognitive science, ethology, and evolutionary biology, an issue to which I will return in a later section. Here, it may suffice to mention that the principles underlying perception must not only be appropriate with respect to relevant properties of the external world but also be functionally adequate, i.e. they have to fit into the entire perceptual and cognitive architecture.

\subsection{The Unit of Analysis}

The detrimental impact of common-sense conception on perception theory stems not only from intuitions about the "truthfulness" and adaptive usefulness of perception but is as much due to our ordinary intuitions about the architecture of our mind. In fact, the entire idea of "architecture of our mind" plays almost no role in our ordinary thinking about mental phenomena. Common sense tells us that it is our integral self, which is in direct contact with the world, and no brain, no intermediate substrate and no properties of whatever happens in the body between the sensory stimulation and the percept figure in its ordinary accounts. In our ordinary conceptions of perception, we discount all the processes that occur between the distal

be regarded as the "true" or "proper" antecedents of the sensory input (aside from heuristic purposes and our ordinary or meta-theoretical talk, in which such inquiries are inevitably embedded) (cf. Mausfeld 2002). 
causes and the percept. Accordingly, we are convinced that we are in direct contact with the world. However, the impression of the unity of our mental life and the conviction that our mind is an integral whole are core - and functionally most important - achievements of our brain. These achievements cannot be taken to convey to us a conception of the underlying functional architecture. In fact, the theoretical picture of the functional architecture of our mind that is emerging in cognitive science, and whose origin can be traced back to Plato and Aristotle, stands in marked contrast to our ordinary conceptions. According to our theoretical insights, we can compare our mind with a huge orchestra in which a plenitude of different instruments act together to yield all our mental achievements. What we can consciously experience is only the sound of the orchestra of our mental faculties working as a whole. We have, however, no experiential access by which we could identify which instruments make which contributions and the precise way in which they act together.

Because ideas about a "functional architecture of our mind" practically have no place in our ordinary conceptions of perception, our ordinary conceptions cannot but to regard the integral person as their unit of analysis. Common-sense-based conception of perception has no need to distinguish between the contributions of different subsystems, and thus between the output of a specific subsystem and the potential uses it is put to by other systems. Accordingly, they tend to identify the output of a specific system, namely the perceptual system, with the results of the functioning of the entire orchestra of mental subsystems, including interpretative ones used for the pragmatics of referring. Due to the fact that in our acts of perceiving, we, i.e. a person, refer to things in the world, reference and ensuing notions play a crucial role in our ordinary conception of perception.

Scientific enquiries of perception, however, have to pursue a different path, which is dictated by their specific explanatory purposes. Perception theory regards as its unit of analysis a specific subsystem of our mind and attempts to identify the abstract internal principles on which its achievements are based by abstracting away from the contributions of other systems of the mind. ${ }^{9}$ Once we regard, in perception theory, a specific subsystem, namely the perceptual system, as the unit of analysis, the notion of reference becomes void of meaning. Consequently, the notion of "reference" and related notions such as "perceptual error", "veridicality", or "proper function" have no place in explanatory accounts of the functioning of the perceptual system, and there are no explanatory lacunae in perception theory to be

\footnotetext{
${ }^{9}$ It is a matter of course that the assumption that the perceptual system qualifies as a subsystem of the brain that can, by standard methodological practices of idealisation and abstraction, be studied in isolation in no way implies the denial of dependencies with other systems. With respect to rational enquiry, the question is not how in reality things are related to each other. The nature and functioning of the perceptual system is related to and dependent on various aspects of reality such as its phylogenetic development, on the metabolic system, the immune system, or a great variety of other internal computational systems, or on the physics of the brain. The question rather is what constitutes an appropriate level of idealisation for successful explanatory frameworks of perception.
} 
filled by introducing these notions. Particularly the notion of "perceptual error", which is a distinguishing element of our ordinary discourse about perception, is of no avail for perception theory (cf. Mausfeld 2002). As already Helmholtz (1855, p 100) emphasised, there are no "errors" with respect to the perceptual system's own principles: "The senses cannot deceive us, they work according to their established immutable laws and cannot do otherwise. It is us who are mistaken in our apprehension of the sensory perception". Notions, such as "error" or "reference", pertain to a different level of analysis, e.g. that of a person, not to the level of a specific subsystem. ${ }^{10}$ The pre-occupation with "perceptual illusions", which is still characteristic for large fields of perceptual psychology and philosophy of perception, indicates the extent to which perceptual research is still driven by common-sense conceptions of perception. Notions of "perceptual errors" have greatly impeded theoretical progress in perception theory and fall back behind clarifications that have been achieved some 100 years ago, and since then have been again and again explicitly expressed by Helmholtz, Hering, Koffka, Köhler, and many other perceptual psychologists.

Apart from the aims of scientific inquiries, the intuitions that characterise our ordinary conception of perception are, needless to say, appropriate and functional. Our ordinary intuition that perception ties us directly to the categories of the world is itself a core achievement of our brain. It becomes deeply misleading only when we employ it in perception theory. Once we decide to theoretically understand, in the context of the natural sciences, the abstract principles by which a specific biological system, the brain, generates its meaningful categorisations and brings forth its perceptual achievements, we have to follow the same kind of methodological principles that we pursue in the case of other objects of nature. This stance, however, which is metaphorically illustrated in Fig. 3, appears to us highly alien and unmotivated from our ordinary intuitions about perception, and thus poses almost insurmountable difficulties for developing appropriate theoretical accounts of perception. With respect to physics, it is well known that its entire history can be read as a continuous quarrel with common-sense intuitions. What holds for physics, even stronger holds for perception theory. We are held captive by the way perception appears to us. Accordingly, pictorial and similarity-based conceptions of perception are so deeply entrenched in our conception of the world and our

\footnotetext{
${ }^{10}$ Most of the currently prevailing philosophical accounts of perception assume that percepts have truth conditions and that the "meaning" of a percept should be conceived in terms of its alleged reference to the external world, i.e. in terms of the conditions under which a corresponding proposition is true. Such accounts rest on some kind of local mapping conception of perception and remain within the confines of common-sense conceptualisations of perception. Whatever their philosophical merits may be, they are of no interest to perception theory.
} 


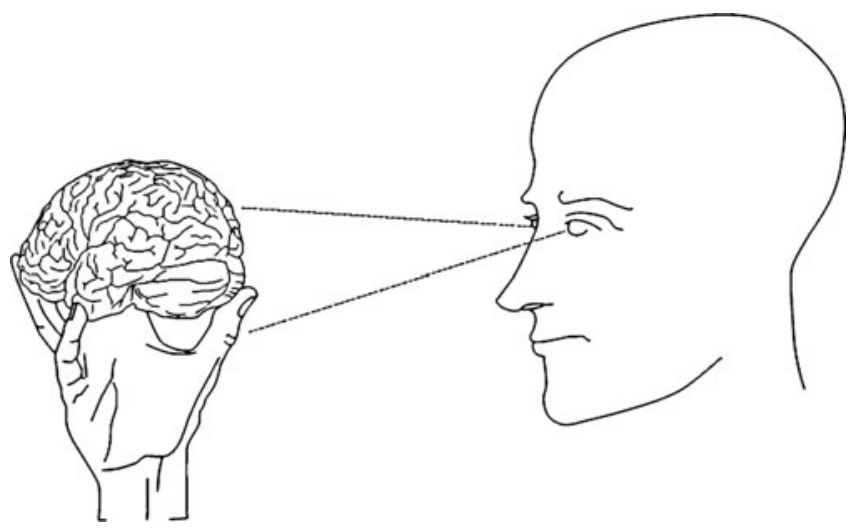

Fig. 3 The target of enquiry for perception theory: the perceptual system regarded as a biological object

interaction with it that it is hardly surprising that they exercise a continuous impact on perception research. ${ }^{11}$

With respect to mental phenomena, we are convinced that our ordinary intuitions about the functioning of the mind were basically correct and that we had some kind of privileged access to the way perception works. However, it is an essential part of the functioning of our brain that it does not allow us immediate introspective insights into its principles. It is part and parcel of the functional architecture of our brain that it almost completely hides the functioning of the perceptual system

\footnotetext{
${ }^{11}$ Also in philosophy of perception, conceptions prevail that are based on philosophically sophisticated varieties of the intuitions underlying naïve realism, such as "critical realisms", or "scientifically informed realism". They are often accompanied by some kind of metaphysical materialism, epistemological reductionism, and the idea that the "meaning" of a percept is determined by its reference to the external world. In contradistinction to other varieties of philosophical realism, structural realism (Russell 1927, cf. also Maxwell 1970; Worrall 1989; Ainsworth 2009) radically dispenses with any kind of realist common-sense intuitions, while still preserving our natural preferences for a realist conception of the world. Structural realism is based on the theoretical observation that from the structure of our perceptions we can "infer a great deal as to the structure of the physical world, but not as to its intrinsic character" (Russell 1927, p 400), and argues that our epistemic access to the external world is restricted to its structural aspects. Such an account is consonant with sign-conceptions of perception. If correspondingly also our epistemic access to the nature of perception is confined to purely structural aspects, qualia by their very nature will escape a deeper explanation in terms of the conceptual frameworks of natural science. Non-structural aspects of perception would then be as much beyond the limits of scientific inquiry as are the non-structural aspects of the physical world, i.e. the "thing in itself". Descartes noticed this and emphasised that the character of qualia, such as the sensation of redness, is not intelligible for us by the rational means by which we investigate the causal structure of the nonexperiential world. Qualia are, in Descartes' formulation, "ordained by nature". Nature has chosen that "certain corporeal events should be "flagged" for us in certain way". But "there is, for Descartes, no scientific explanation of the flagging system which actually obtains" (Cottingham 1986, p 140).
} 
from our conscious experience. What we consciously perceive is only the end product of the perceptual system. Only by the standard methodological procedures of the natural sciences can we attempt to identify the principles on which perceptual achievements are based.

\section{The Standard Model of Perception}

The development of an appropriate theoretical account of the internal principles that underlie our perceptual achievements is, unsurprisingly and almost inevitably, impeded by the obstructive influences of common-sense intuitions. Variants of naïve realism and ensuing conception are still prevailing as a tacit background assumption in perceptual psychology, albeit often concealed by technically sophisticated formulations. These conceptions have affiliated with the idea, originating from sensory physiology, that the sense modalities can be considered as the natural starting point and as the natural units of analyses for perceptual psychology. As a result, the nature of the perceptual system predominantly has been carved along the lines of sensory input channels, and perceptual psychology has been organised in terms of elementary perceptual attributes. Corresponding ideas have condensed in what has become the Standard Model of Perception, as depicted in Fig. 4.

The Standard Model of Perception, in its many guises, is grounded on a distinction between sensations, as the "raw material" of experience, on the one hand, and perceptions, which are typically conceived of as referring to objects in the external world. According to this model, the process of perception can essentially be described as subsequent stages of "information" processing by which the sensory input is transformed into the meaningful categories and distinctions, namely the "perceptions", whose meanings derive from what they refer to in the external world. More specifically, it is presumed that the sensory input provides information about elementary sensory qualities, such as colour and motion, and that by so-called lower

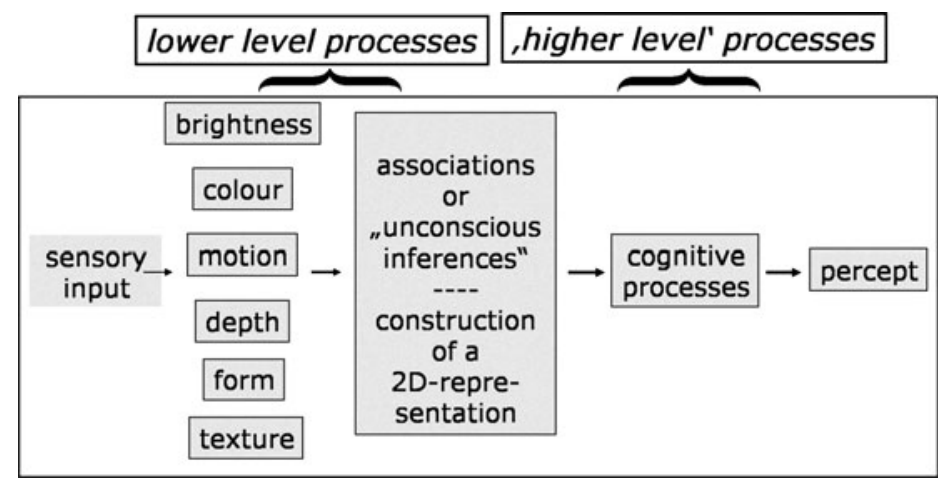

Fig. 4 The standard model of perception 
level processes these elementary qualities are glued together by some associative or inferential machinery. ${ }^{12}$

The remaining gap to the percept is then allegedly bridged by what is usually referred to as "cognitive processes" or "higher-order" processes, which are assumed to account for everything that cannot be explained by the so-called lower-level processes. The Standard Model presumes that the biologically given conceptual endowment underlying perception is confined to a rather thin set of sensory concepts from which all other concepts have subsequently to be derived by some inductive or associative machinery. ${ }^{13}$

The Standard Model is already deeply flawed on conceptual grounds. Its key conceptual flaw is that it is based, in line with our common-sense intuition about perception, on a fatal conflation by which the output of the system under scrutiny is mistaken for its input. The Standard Model surreptitiously borrows semantic distinctions, such as "perceptual object", "surfaces", "shadows", or "illumination", tacitly from the output of the perceptual system and uses them for a description of the input (particularly when the Standard Model is supplemented by an inverse optics approach of "recovery of world structure"). By its very conceptualisation of perception, the Standard Model dodges an essential task of perceptual research, namely the identification of the internal conceptual structure of perception and the symbolic objects to which the computational procedures of the perceptual system apply. Characteristically, the Standard Model shirks the explanatory task to come to terms with the most fundamental theoretical notion, namely that of a "perceptual object", by deriving this notion from an allegedly "corresponding" notion of an "external world object" and by placing the explanatory burden on experience and some inferential machinery. The Standard Model trivialises what can be regarded as the Fundamental Problem of Perception Theory, namely the identification of the principles, by which the perceptual system generates, given a specific physical spatio-temporal energy pattern as input, an output that is organised in terms of semantic categories. Accordingly, the problem of perceptual semantics has not even been recognised by the Standard Model

\footnotetext{
${ }^{12}$ Corresponding ideas are, however, profoundly inadequate already on conceptual grounds (as has also been emphasised by gestalt psychology). Insights into what can be achieved by inductive procedures made clear that no general inductive machinery, however powerful, can derive from the sensory input, and thus, more generally, from experience, the kind of internal conceptual structure that is explanatorily required (unless it is already itself based on a conceptual structure as powerful as the one to be inferred). However powerful the inductive machinery is assumed to be, there is no way to arrive at symbolic objects that are logically more powerful in the sense that their structure is not expressible in the logical language in which we describe the bases of the inductive procedure (cf. Fodor 1980). Since essentially no perceptual object or attribute of our perceptual system is definable in the logical language by which we describe the physical energy pattern that constitutes the sensory input, the internal structure underlying perceptual meaning cannot be attained by inductive procedures (unless we surreptitiously describe the input in terms of the yet-to-be-explained output categories).

${ }^{13}$ Apart from being unmotivated from the point of evolutionary biology, this presumption, however, does not derive from empirical evidence but rather expresses a deeply entrenched epistemological prejudice, namely the empiricist conception of the mind, which itself can be regarded as a philosophically refined expression of our ordinary pre-conceptions about mental achievements.
} 
as a serious theoretical challenge because it is concealed precisely by one of the eminent achievements of our brain, namely the externalisation of its own semantic categories into what we regard as the external world. ${ }^{14}$

In the history of perceptual psychology, the strongest critique of the Standard Model had been advanced by Gestalt psychologists, on the basis of accumulating empirical evidence. They furthermore recognised that the Standard Model's emphasis on issues of processing results from mistaking the explanatory task of neurophysiology for the explanatory task of perceptual psychology, and thus from conflating different levels of analysis. However, the Standard Model is, despite its utter conceptual and empirical inadequacy, is still prevailing in perceptual research, where it is mostly regarded as a truism (cf. Mausfeld 2002, 2003a, 2010b). ${ }^{15}$

Fortunately, however, more appropriate conceptions have emerged during recent decades, in a convergence of empirical findings and theoretical ideas from ethology, research with newborns, perceptual psychology, and cognitive science. These conceptions, which were foreshadowed by Gestalt psychology and theoretical insights during the seventeenth century, provide a theoretical framework for perception that radically deviates from the Standard Model. Already at the beginning of the last century, the empirical and theoretical evidence in support of corresponding ideas was enormously rich. But only after advances in the computational sciences provided a

\footnotetext{
${ }^{14}$ The conceptual defects of the conceptions underlying the Standard Model had already been clearly identified in the seventeenth century. By corresponding enquiries, it became evident that the problem of perceptual meaning cannot be resolved by deferring the explanatory duty to the sensory information. Even the core notion of a "perceptual object" cannot be derived, by whatever mathematical machinery, from the sensory input. Hume was well aware of this problem and noted, in his Treatise of Human Nature (Book 1, Part IV, sec II), that the senses "give us no notion of continu'd existence, because they cannot operate beyond the extent, in which they really operate. They as little produce the opinion of a distinct existence, because they neither can offer it to the mind as represented, nor as original... We may, therefore, conclude with certainty, that the opinion of a continu'd and of a distinct existence never arises from the senses." Therefore, it has generally been noticed since the seventeenth century that there is an explanatory gap to be filled.

${ }^{15}$ The Standard Model is deeply rooted in the behavioristic tradition. One can hardly overestimate the impact and longevity of behavioristic thinking. Although it is concealed by the cognitivist jargon it has adopted, its hallmarks are easily discernible, namely an underlying empiricist conception of mind, an inductivist and empiricist epistemology, and a bias favouring investigations of input-output relations at the expense of inquiries into internal principles. Miller (1979) observed two decades after the beginning of the so-called cognitive revolution: "What seems to have happened is that many experimental psychologists who were studying human learning, perception, or thinking began to call themselves cognitive psychologists without changing in any obvious way what they had always been thinking and doing - as if they suddenly discovered they had been speaking cognitive psychology all their lives. So our victory may have been more modest than the written record would have led you to believe". In a similar vein, Fodor (2003) noticed: "In fact, though, practically all experimental psychologists and philosophers of mind continue to be behaviourists of one kind or other. They have just ceased to notice that they are". Even worse, the impact of behaviourist pre-conceptions is mostly not regarded as a flaw but claimed as a virtue. Roediger (2004) gives an unusually explicit statement of this attitude: "Behaviorism is alive and most of us are behaviorists".
} 
new conceptual apparatus, could these ideas be taken up and further explored in a fruitful manner.

Although the theoretical picture that is emerging from corresponding enquiries is still very skeletal, it has already yielded intriguing results with respect to a range of significant phenomena and has suggested novel and fruitful questions about the internal architecture of perception. It is conceptually and empirically well supported and has considerably gained in explanatory width and depth. Our confidence in the appropriateness of the emerging theoretical framework furthermore is, as always in the natural sciences, fortified by the fact that it is yielded by a theoretical convergence of quite different disciplines. All the same, these developments have not yet gained wider acceptance because they are grossly at variance with our common-sense conceptions about perception.

\section{Fundamental Aspects of the Functional Architecture and of the Conceptual Forms Underlying Perception}

The Fundamental Problem of Perception Theory originates from the fact that the output of the perceptual system, namely meaningful categories, is vastly underdetermined, as it were, by the sensory input, namely physico-geometric energy patterns. Approaches that are in line with the ideas underlying the Standard Model tend to gravely underrate the explanatory gap involved because they confound output and input categories. As a result, these approaches underestimate the quantity and complexity of the biologically given conceptual endowment of the perceptual system explanatorily required to account for its achievements. They rather exhibit a preference for ascribing to the system only a meagre set of sensory concepts. ${ }^{16}$ While corresponding ideas are suggested by common-sense intuitions, they prove to be profoundly inadequate in the context of the Fundamental Problem of Perception Theory.

The theoretical framework that has been emerging during the last decades from a convergence of different disciplines acknowledges, in line with a growing body of experimental evidence, that we can only deal in an explanatorily adequate way with the Fundamental Problem of perception theory when we are willing to subscribe to the perceptual system a biologically given conceptual structure that is rich enough to account for its core perceptual achievements. Of course, the theoretical picture whose contours are presently emerging at the horizon is still faint and inevitably speculative. I cannot evaluate here the abundantly rich empirical evidence that speaks in favour of this picture. Since my agenda here is primarily programmatic in

\footnotetext{
${ }^{16}$ If we regard the perceptual system, in a rather loose sense, as a computational system, we have to explicitly specify the data formats on which computational processes are based. Approaches that follow the lines of the Standard Model of Perception tend to confine the type of data formats that are part of the biological endowment of the perceptual system to those that can be defined in terms of elementary sensory features.
} 
character, I will attempt to venture some ideas about the nature of conceptual forms that appear to me theoretically motivated and empirically warranted on the bases of what can be distilled from experimental findings in various domains. Ethology, perceptual psychology, and research with newborns and babies have provided, notably in more recent studies, a rich and fruitful basis for theoretical speculations about the nature of the conceptual forms underlying perception. ${ }^{17}$

The available evidence suggests that the perceptual system is biologically endowed with an exceedingly rich set of complex conceptual forms in terms of which we perceive our "external world", such as "surface", "physical object", "intentional object", "event", "potential actor", "self", or "other person", with associated attributes, such as "colour", "shape", or "emotional state", and their appropriate relations, such as "causation", "intention", or, in the case of "event", "precedence", "beginning", "end", or "subsequence". These conceptual forms cannot be derived, by whatever mechanisms of inductive inference, from the elementary sensory predicates delivered by the senses. We thus have to assume that the perceptual system is biologically endowed with a rich conceptual structure in terms of which the signs delivered by the senses are exploited.

At the core of this theoretical picture is the idea that, in more complex organisms, the sensory input serves as a kind of sign for the activation of biologically given conceptual forms, which determine the data format of the computational processes involved. The conceptual forms, say for "surface", "food", "enemy", or "tool", cannot be reduced to or inductively derived from the sensory input but have to be regarded as part of the specific biological endowment of the organism under scrutiny. ${ }^{18}$ In order to account for the relation between the sensory input and the irreducible and complex perceptual concepts that constitute the output of the perceptual system, it is useful to distinguish at least two levels of abstraction ${ }^{19}$ with respect to the functional architecture of the perceptual system, namely a

\footnotetext{
${ }^{17}$ These speculations can only be regarded as fruitful to the extent that they contribute to a core task of perception theory, namely the development of an explanatory framework of sufficient explanatory depth and explanatory width for a range of basic phenomena. Due to this requirement, regarded as a matter of course in the natural sciences, any confidence in corresponding theoretical speculations will not be based on isolated experimental findings but rather on a deeper convergence of quite different disciplines on a joint theoretical perspective.

${ }^{18}$ It is important to be aware that these linguistic appellations of the conceptual forms of the Perceptual System are only makeshift descriptions of non-linguistic entities (which are, of course, further shaped by the properties of subsequent linguistic and interpretative systems).

${ }^{19}$ Employing different levels of abstraction does not eo ipso imply any ontological commitments as to the physical or biological organisation of the system under scrutiny. However, additional arguments, e.g. from evolutionary biology, can be advanced in favour of the assumption that there are in fact different and to some interesting degree modular subsystems involved. Therefore, I identify the required levels of abstraction with actual (idealised) subsystems (and the logical language by which we describe the functioning of a system with the logical language in which the actual operations of the system can be expressed).
} 


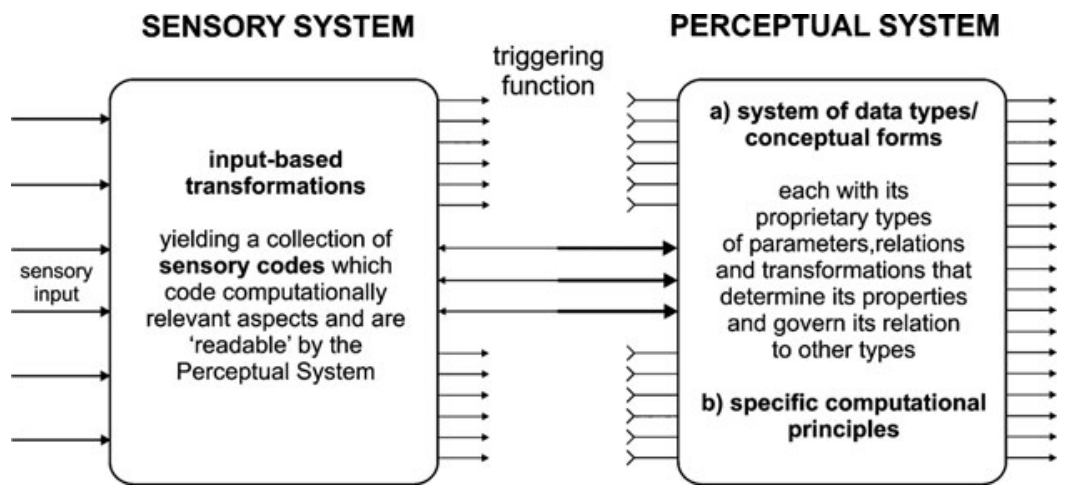

Fig. 5 Functional architecture of the perceptual system

Sensory System, on the one hand, and Perceptual System, on the other hand (as illustrated in Fig. 5). ${ }^{20}$

The Sensory System, understood in the technical sense used here, deals with the transduction of physical energy into neural codes and their subsequent transformations into codes that are "readable" by and fulfil the structural and computational needs of the Perceptual System; we can refer to these codes as "cues" or "signs". ${ }^{21}$ For each given sensory input, all codes that are computable from this sensory input by the available computational apparatus and that can be "read" by the Perceptual System are simultaneously provided at the interface to the Perceptual System. Thus, the Sensory System can be understood as providing a specific subset of the (infinitely many) relations etc. of the sensory input, namely that subset that can be computationally exploited by the Perceptual System in terms of its conceptual forms. The coding properties of the Sensory System are highly

\footnotetext{
${ }^{20}$ I keep the term "perceptual system" for loosely referring to the entire modular system of perception, which includes the Sensory System as well as the Perceptual System as characterised here. It is important to note that the distinction between a Sensory System and a Perceptual System as employed here is entirely different in character from the sensation-perception distinction as introduced by Spencer, James, Wundt, or Helmholtz, which refers to an alleged hierarchy of processing stages, in terms of the same data formats, by which the sensory input is transformed into "perceptions". On this highly influential distinction, sensations are regarded as the "raw material" of experience, while perceptions are typically conceived of as referring to objects in the external world. Sensations and perceptions were generally held to "shade gradually into each other, being one and all products of the same psychological machinery of association" (William James 1890/1983, Ch. XIX), or of some other inferential machinery.

${ }^{21}$ Note that for the generation of "signs" by the Sensory System, it is irrelevant whether the sensory input has been causally generated by a real object, by a picture of this object on a computer screen or by an appropriate stimulation of nerve cells. Hence, the "external objects" in terms of which we describe, in our ordinary discourse, the external world, do not figure in explanatory accounts of perception.
} 
idiosyncratic, ${ }^{22}$ with respect to our physical descriptions of the external world, and often strongly deviate from our ordinary intuitions and expectations.

The distinguishing feature of the Sensory System is that the data formats on which its computations are based can be entirely defined in terms of the elementary physico-geometrical language in which we describe the geometrically organised sensory input, i.e. in terms of lines, gradients, texture, contour, and luminance. The Perceptual System, on the other hand, contains the rich set of complex conceptual forms (such as "perceptual object", "surface", "food", "enemy", "tool", and "causal event") that are not definable in terms of the conceptual forms of the Sensory System or reducible to them. These conceptual forms thus define the entities or abstract objects for the internal computations of the Perceptual System. They are the basis for our "perceptual ontology" and thus constitute the realm of "perceptual objects". We can conceive of the Perceptual System as a self-contained system of "perceptual knowledge", an "integrated complex represented in the mind" which pertains to "a specific domain of potential fact, a kind of mentally constructed world" (to borrow Chomsky's 1981, p 6, description of a different subsystem of the mind). We can refer to what is coded in the structure of its conceptual forms as the "internal semantics" of perception. According to the architectural conception proposed here, core aspects of most types of the explanatorily required build-in "knowledge" as assigned to "core knowledge systems" by Spelke (2000) and Spelke and Kinzler (2007) or to "conceptual-intentional systems" in the minimalist programme of linguistics have to be imputed to the Perceptual System already. At the core of this theoretical conception is the idea that, in more complex organisms, perception is based on an internal computational organisation in terms of abstract data types or conceptual forms; and that the sensory input serves as a kind of sign for the activation of these biologically

\footnotetext{
${ }^{22}$ With respect to sensory systems, no one will doubt the obvious fact that their coding properties cannot simply be derived from alleged adaptive requirements. For instance, we have no problems in accepting the idea that the qualitative experience of colours, say the experience of a certain red, and also their structural relations, say the fact that red is perceptually nearer to blue than to green, cannot be derived, by however sophisticated mathematical transformations, from the sensory input or from external physical regularities. Rather, we regard these properties as being essentially codetermined by internal physical and neural constraints, i.e. by constraints that are idiosyncratic and contingent with respect to any adaptive coupling of the organism to its environment. Oddly enough, the same considerations receive less credibility in the case of more complex properties of our perceptual system. However, the structure of the conceptual forms, on which the computations of the perceptual system are based, will, in its evolutionary development, most likely be essentially co-determined by constraints that stem from the fact that only certain physical and computational channels were open as feasible evolutionary paths. The conceptual forms of the perceptual system must not only be adequate with respect to the external world (however one understands such a requirement); they must also be computationally adequate, i.e. they have to fit into the entire computational architecture. Any attempt to understand the nature of the conceptual forms of the perceptual system merely from the perspective of an adaptive coupling to the physical world will most certainly be doomed to fail. Apart from the fact that it is based on a misguided and defective research heuristics, it grossly underestimates the rich internal constraints that arise within the computational architecture of the biological system under scrutiny.
} 
given conceptual forms, which constitute the data formats of the computational processes involved. This conception is metaphorically illustrated in Fig. 6.

The output of the Perceptual System consists of structured entities that are generated by its proprietary computational machinery and that represent legible instructions for subsequent systems by which interpretations in terms of external world properties are rendered. The instructions provided by the Perceptual System are exploited and "interpreted" by subsequent interpretative systems (which include belief systems and systems comprising non-perceptual knowledge about the world), systems for imaginations, or systems by which the phenomenal percept is generated. Thus, the Perceptual System provides, at its interfaces to higher-order interpretative systems, options for exploiting activated conceptual forms in terms of the conceptual forms and computational requirements of these higher-order systems. A demarcation between the Perceptual System and higher-order interpretative systems is much more difficult to achieve than between the Perceptual System and the Sensory System. Hence, the line of demarcation depends to some extent on theoretical and meta-theoretical preferences. At any rate, operations that enable us to refer to properties of our external world and thus the computational and conceptual apparatus for externalising, as it were, the conceptual forms of the Perceptual System have to be assigned to higher-order interpretative systems rather than to the Perceptual System itself.

The conceptual forms of the Perceptual System can be understood as those data formats underlying its computations that are "visible" at its interfaces with subsequent systems and thus can be "read" by those systems. However, the output of the Perceptual System cannot be expected to be fully expressible in terms of higher-order interpretative and phenomenal systems. Rather, it can be used by other

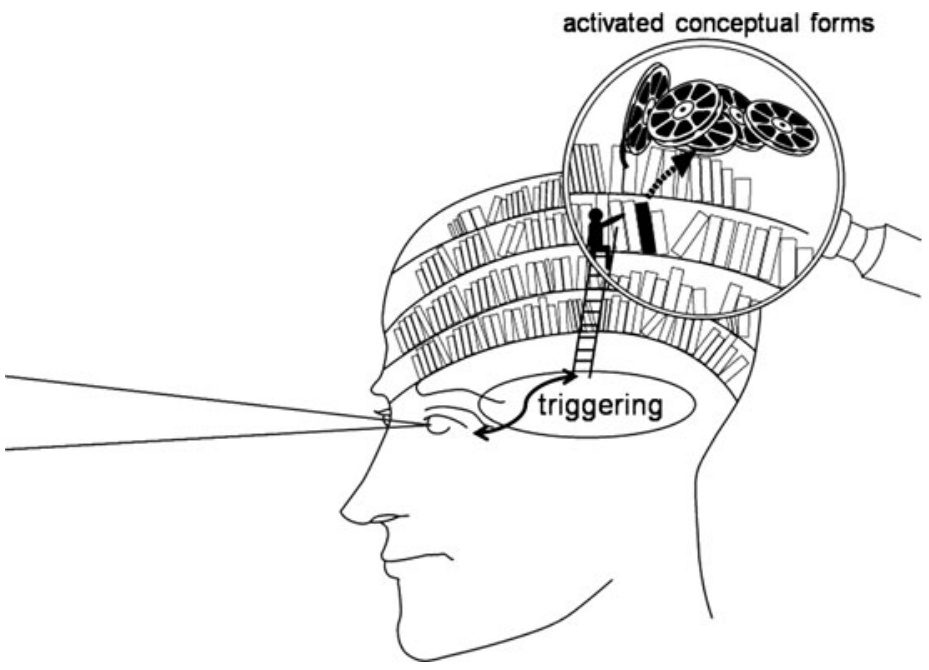

Fig. 6 Perception as triggering of biologically given conceptual forms 
internal systems, such as ones subserving motorial purposes, and remain partly phenomenally silent, or un-interpreted by higher-order interpretative systems.

The conceptual forms of the Perceptual System can be investigated with respect to various aspects. I will briefly address structural aspects, i.e. aspects of their logical form, their dynamical role in structure-building computational processes within the Perceptual System, and the question whether such conceptual forms can be assigned some biological plausibility from an evolutionary point of view.

\subsection{Structural Aspects of Conceptual Forms}

As to the structural aspects, I have to confine myself to a few more intuitive remarks guided by conceptual considerations because our current understanding is too poor as to make any attempts at a more formal characterisation premature (this situation is exacerbated by the problem that, for well-known reasons, conceptual forms cannot be defined by the extensional notion of a set of objects). On the assumption that the biological system under scrutiny can be regarded as a computational one, we can conceive of the conceptual forms of the Perceptual System as abstract structures, each of which has its own proprietary types of parameters, relations, and transformations that govern its relation to both other conceptual forms and sensory codes. The values of the free variables of a conceptual form in general will not be and, for subsequent computational processes, need not be - exhaustively specified by the activating input.

Empirical evidence furthermore indicates that the conceptual forms of the Perceptual Systems can be classified into different types. Types are sets of computational objects with uniform behaviour which usually can, in the meta-language we employ, be named (e.g. "surface"). Types are associated with constants, operators, variables, and function symbols by which they code the constraints on the computational processes associated with certain "perceptual objects". For instance, computational processes associated with "perceptual objects" of the type "living animal" pertain to internal causal analyses in terms of "hidden powers" and "essences" of those types of objects; computational processes associated with "perceptual objects" of the type "mykind", pertain to causal analyses in terms of "mental states". The hierarchy of types constitutes the build-in knowledge about entities, situations, actions, perspectives etc. of our perceptual world. Conceptual forms and their associated computational processes provide a "grammar of vision", as it were. "Employing this "grammar of vision" - largely innate - higher animals are able to "read" from retinal images even hidden features of objects, and predict their immediate future states, thus to "classify" objects according to an internal grammar, to read reality from their eyes (Chomsky 1975, p 8). From the theoretical perspective propose here, there is no difference of principle, with respect to the fundamental mode of operation of the perceptual system, between the perception of material properties and the perception of, say, mental states of others. 
Conceptual forms can be regarded as semantic atoms of the internal semantics of perception. They express the core semantics of minimal meaning-bearing elements. Conceptual forms generate semantic and syntactic molecules and constitute, together with the computational apparatus of the Perceptual System, the combinatorial language of perception, as it were. The Perceptual System can be expected to have its own computational and generative principles for the structure-building processes by which systematically related patterns of activated conceptual forms are generated, in particular principles that pertain, e.g. to an evaluation metric, to the satisfaction of internal constraints, or to more global coherence constraints. For instance, perceptual phenomena suggest that it possesses a rather idiosyncratic evaluation metric (as mirrored in phenomena traditionally discussed under the heading of "cue integration") and likewise idiosyncratic principles by which it glues together fragmented structures of activated conceptual forms into a globally more coherent structure (as witnessed, e.g. by phenomena pertaining to so-called impossible objects, or to the "dual nature" of picture perception). Conceptual forms are organised in highly hierarchical structures. They comprise, as part of their structure, relational parameters that act as computational synapses, as it were, to other computational forms and whose values define the strength of the intrinsic connections between conceptual forms. Accordingly, conceptual forms build systematically connected packages whose nature expresses the kind of built-in "knowledge" of the Perceptual System; also the activated conceptual forms offered at the interface to higher-order systems come in systematic bundles.

\subsection{Biological Plausibility of Conceptual Forms}

Because of the highly abstract and logically complex nature of conceptual forms, adherents to the Standard Model tend to regard them as biologically implausible entities and thus as explanatorily unwanted and dubious concepts for perception theory. However, which kind of theoretical notions are biologically plausible and which are not cannot be assessed in an a priori way but only by the explanatory success of an entire theoretical framework. Within the framework of evolutionary biology, independent lines of theoretical arguments can be discerned by which the biological emergence of abstract data types or conceptual forms can be motivated. These arguments are based on the observation that modularity, in some form or the other, presumably is, at all levels of biological organisation, the basis for the evolvability of complex systems and a driving force in their evolution (e.g. Kirschner and Gerhart 1998). Accordingly, we can also expect computational systems, such as those subserving perceptual achievements, to exhibit a high degree of modularity with respect to the data structures over which the corresponding computations are defined. An evolutionary increasing differentiation or specialisation of computational subsystems mediating between the front end side of sensory transduction, on the one hand, and the motoric effector side, on the other, will accordingly yield an increasing number of interface problems that the system has to solve as a result of its increasing 
modularisation. For organisms with the simplest types of nervous systems, we can observe that the integration of different sensory channels is kept to a minimum, as required for instant motor control and action selection. An example is Cubozoans or box jelly fishes, which possess 24 eyes of 4 different types, where each type presumably serves a different adaptive function (Nilsson et al. 2005; Jacobs et al. 2007). Computationally more thorough degrees of informational or conceptual integration in organisms with multiple sets of eyes can be found at higher evolutionary levels of neural organisation (e.g. in jumping spiders, Harland and Jackson 2000). For organisms with more complex nervous systems, comparative neurobiology and ethology offer many findings that indicate that the same type of sensory input can be exploited by different computational subsystems, each having its proprietary data type, apparently without being conceptually integrated in a more thorough and comprehensive way (cf. Tinbergen 1951, classical example of the egg-roll behaviour of the grey goose, which presumably employs, depending on context, two disparate and apparently non-integrated concepts with different triggering conditions, namely "egg outside of the nest" vs. "egg inside the nest"). Although our current knowledge of the evolutionary emergence of abstract data types/conceptual forms (also referred to as "symbolic representations", e.g. Gallistel 1998) is still wanting, we can infer some general features from synchronic investigations of extant species as well as from insights into the role of internal (i.e. non-adaptational) physical principles in evolutionary processes (e.g. Kirschner and Gerhart 1998; Kitano 2004; Wagner et al. 2005; Müller 2007; Goodwin 2009; Newman and Bhat 2009), principles that presumably have their analogues on computational levels of biological systems. On the assumption that complex perceptual systems are computational systems and that modularity, with respect to corresponding data formats, is a driving force for their evolutionary differentiation, abstract data types will likely emerge as a consequence of internal computational and physical requirements of the functional organisation of the evolving system. In the course of an increasing evolutionary differentiation of the neural substrate that processes the information from various sensory channels, the system is increasingly faced with the functional demands of computationally tying together different subsystems by a joint data format that is sufficiently abstract for an integration of the information provided by different subsystems. While conceptually shallow interfaces may suffice in simple systems, in which different subsystems can be closely tied to single adaptive functions, such as recognition of prey, of mates, or of conspecific rivals, more complex systems would yield an increasing number of executive conflicts at the motorial interface unless they exhibit an appropriate degree of inferential and conceptual integration between different subsystems. In this sense, the biological tendency for an evolutionary increasing amount of modularisation spurs and enforces, with respect to computational systems, an increase in data abstraction and hence the development of conceptual forms. Because a data type together with its associated operations can be regarded as a computational module, the availability of conceptual forms themselves can be regarded as an extreme variant of modularity.

If, as seems not unreasonably to conjecture, conceptual forms are evolutionary brought forth by internal computational requirements, their specific forms are not 
solely constrained by the adaptive requirement of coupling the organism as an entirety to its environment. Rather, the specific structure of the conceptual forms underlying perception will most likely be essentially co-determined and shaped, within the apparently rather broad latitude of design options that are left open by global adaptational restrictions, by powerful internal constraints that arise during the evolutionary development of a system of this complexity. Accordingly, the conceptual forms on which the computations of the Perceptual Systems are based have their own properties, which can be rather surprising when viewed exclusively from the perspective of an adaptive coupling to the external world. In fact, the most complex perceptual achievements, such as seeing invisible properties of objects (e.g. material qualities), intentional properties of objects (e.g. tools), or mental states of others, only became possible by evolutionary decoupling the output of the Perceptual System from the information provided by the sensory codes and by furnishing the Perceptual System with its proprietary types of abstract data types or conceptual forms.

\subsection{Dynamic Aspects of Conceptual Forms}

Finally, I address some dynamical aspects of the structure-building computational processes of the Perceptual System. The computations within the Perceptual System are instigated, via a triggering function between the Sensory System and the Perceptual System, by the sensory codes caused by a given sensory input. ${ }^{23} \mathrm{We}$ can intuitively think of the triggering functions as an interface function that takes specific sensory codes as an argument and calls conceptual forms ${ }^{24}$ (cf. Fig. 5).

\footnotetext{
${ }^{23}$ Corresponding intuitions had already been formulated in the seventeenth century. They found their elaborate expression notably by Cudworth (1731), who conjectured that conceptual forms "are excited and awakened occasionally from the appulse of outward objects knocking at the doors of our senses" and that "sense is but the offering and presenting of some object to the mind, to give it an occasion to exercise its own inward activity upon". The Perceptual System "is enabled as occasion serves and outward objects invite, gradually and successively to unfold and display it self in a vital manner, by framing intelligible ideas or conceptions within itself of whatsoever hath any entity or cogitability" (cf. Mausfeld 2002).

${ }^{24}$ The explanatory need for a triggering function as a function mediating between computational systems of different logical expressive power has been clearly recognised by Descartes. He was aware that any reference to "external objects" in sign conceptions of perception would lead, within scientific enquiry, to conceptual incoherences. In his attempts to provide, in entirely naturalistic terms, an explanatory framework for bridging the explanatory gap associated with the Fundamental Problem of Perception, Descartes (1642/1985) formulated a purely internalistic version of a sign theory of perception. Yolton $(1984,1996)$ referred to Descartes' conception as "inverse sign relation", because for Descartes, the physical motion, as expressed by neural activity, is the sign, and what is signified is what is expressed in the percept (cf. Gaukroger 1990, p 24). For Descartes, there are "two reactions operating in perception: the causal, physiological reaction and the signification reaction" (Yolton 1996, p 74). The significatory or semantic relation "replaces the causal relation between physical motion and ideas, but the representing relation goes, as it were, outward from awareness" (Yolton 1996, p 190). Descartes thus recognised the explanatory need for postulating a "semantic relation" in perception. "The connection between the signs and innate
} 
This intuition, however, is not entirely appropriate because it neglects the dynamic bidirectional aspect of this function by which conceptual forms search, as it were, for satisfying conditions of sensory codes. On the theoretical account proposed here, the sensory codes serve a twofold function. They activate, via the triggering function, appropriate conceptual forms and thus determine the potential data formats in terms of which input properties are to be exploited. Furthermore, they assign concrete values to the free parameters of the activated conceptual forms. Such a description, though, is much too static to capture, in an empirically adequate way, the computational achievements of the Perceptual System. Actually, a satisfactory theoretical account of the structure-building processes within the Perceptual System, by which outputs are generated that are legible to higher-order systems, has to provide something in its explanatory toolkit by which we can cope with the dynamic aspects and the "creative forces" (von Szily 1921) of perception. These aspects pervade the entire realm of perceptual phenomena, although they rarely become phenomenally salient. We can, however, notice them in a more perspicuous way in phenomena pertaining, for instance, to context-dependent reorganisations of part-whole relationships, or to re-conceptualisations of "perceptual objects" and their "parts", and in all situations in which ambiguities or perceptual vagueness occur. Any explanation of such phenomena requires the availability of special computational means and of conceptual forms with structural properties that promote and boost these dynamic properties. Corresponding intuitions about dynamic conceptual forms stretch back to Descartes, Humboldt (with his emphasis on active structure-building "energia"), and Cassirer. Despite the overwhelmingly rich empirical evidence fostering such intuitions, we are still lacking appropriate scientific conceptions for capturing these "active", abductive and dynamic elements of perception in a more precise and explanatory adequate way.

The general theoretical perspective adopted here, which focuses on conceptual forms and their triggering functions, prompts to derive from experimental findings and phenomenological observations some preliminary speculations about potential structural aspects of conceptual forms that yield or support these highly contextsensitive dynamic properties of the Perceptual System. A structural aspect that suggests itself is the option to leave values of parameters undetermined by the input (or to only constrain them to regions of their associated parameter space). It is the presence of un-interpretable elements that makes conceptual forms "computationally active" (in the sense that they are probing, as it were, the available sensory cues), and they remain active as long as they contain such elements demanding

ideas is clearly more intimate than any causal connection would be, for it is the innate ideas that make the signs what they are, whereas effects can never make causes what they are". (Gaukroger 1990, p 25). Descartes' intuitions about this "semantic relation" between what is provided by the senses, on the one hand, and the conceptual forms or "ideas" that yield meaningfully organised percepts were far ahead of anything that could be expressed in terms of the conceptual apparatus available at his time. Unsurprisingly, Descartes' vacillating and tentative usage of terms, due to which he is notoriously hard to interpret, mirrors this lack of an appropriate conceptual framework, as provided much later by computation theory. 
satisfaction. If a triggering function would yield an activation pattern of conceptual forms that completely specifies all values of parameters and satisfies all internal constraints of the Perceptual System, no further structure-building would be required. If, however, constraints are violated or undetermined values of parameters remain, further structure-building by computational processes is required, involving appropriate evaluation functions.

Experimental findings (for striking cases see, e.g. Kellman and Spelke 1983; Craton 1996; Tse 1999) as well as phenomenological observations indicate that the Perceptual System seems to routinely operate semantically with underdetermined conceptual forms. There is every indication that this structural feature is a general property of conceptual forms that extends to subsequent systems. Consider, for example, systems by which phenomenal percepts are generated. From a naïve perspective, one could be tempted to assume that these systems assign a semantically fully and uniquely determined phenomenal percept to the output of the Perceptual System. In fact, however, the ubiquitous phenomena pertaining to vagueness, indeterminacy, or uncertainty indicate that the underlying conceptual forms inherit the underspecifications, with respect to a given input, of the conceptual forms of the Perceptual System. The output of the Perceptual System, at its interfaces to subsequent systems, thus needs not to be semantically determinate or unique but only "good enough" for the semantic needs of the subsequent systems.

With respect to the possibilities of lacking specificity and semantic determinateness, it is useful to roughly distinguish, among the entire spectrum of types of underspecification, global and local aspects. Global aspects of underspecification pertain to an entire perceptual "interpretation", as determined by a systematically related bundle of conceptual forms. Local aspects pertain to features of individual abstracts objects that belong to an activated systematic bundle of conceptual forms. Accordingly, we can speak of globally underspecified (bundles of) conceptual forms and locally underspecified conceptual forms. Local underspecifications, in otherwise globally determined "interpretations", pervade almost all aspects of perception; mostly, the underspecification is never completely resolved or only resolved in the context of specific tasks. In contrast, global types of underspecification are not as prevalent and seem to require the kind of input situations that are associated with phenomena of multiperspectivity.

All forms of underspecification convey constitutive computational advantages to the perceptual system. By postponing disambiguation to higher-order interpretative systems, the Perceptual System can increase its global stability with respect to the superordinate "interpretations" provided at its interfaces to subsequent systems. This protects the system from settling, under insufficient or "impoverished" input situations, on some definite "interpretation" that would have to be changed to an entirely different "interpretation" following a small variation in the input. In addition, and independent of issues of handling impoverished input situations, underspecified conceptual forms boost the potency of generative processes and enhance the conceptual versatility of the Perceptual System. By routinely operating with underspecified conceptual forms, computations can be performed on different 
levels of semantic granularity. The computational advantages of the capability to perform semantic operations over underspecified conceptual forms have been, albeit in different theoretical frameworks, profitably explored in computational semantics (e.g. Pinkal 1996; Pustejovsky 1998).

The theoretical picture underlying this way of framing core issues of perception evidently differs considerably from prevailing ones. It reverses the traditional perspective and approaches perception rigorously from within outwards, as it were. Needless to say that in the light of what is currently at all well understood about fundamental principles underlying perception, the framework adumbrated is inevitably highly speculative. Yet, it appears to me well motivated in the light of forceful theoretical arguments that can be advanced in favour of it, as well as in the face of a great variety of impressive empirical evidence supporting it. Presently, however, only the contours of a theoretical picture have become faintly visible due to the current convergence of different disciplines. More specific ideas and hypotheses can only be ventured in the course of enquiries that systematically explore the purview and fertility of this theoretical framework. Here, I have to content myself with intuitively illustrating the potential fecundity of the proposed theoretical stance. While traditional accounts of perception are fundamentally ill equipped to deal with aspects of meaning, multiperspectivity, and perceptual vagueness, attributing them essentially to "higher-order" cognitive systems, the direction of enquiry outlined above promises a natural way of approaching them as intrinsic properties of the perceptual system proper.

\section{Intrinsic Multiperspectivity}

As mentioned in the introduction, the capacity to employ different mental perspectives with respect to the same stimulus situation pervades all of our mental activity. Once general properties of such phenomena of "multiperspectivity" are explored on a sufficient level of abstraction, it becomes obvious that cognitive science teems with corresponding phenomena, which witness, it seems to me, our cognitive capability to simultaneously handle different layers of perceptual interpretations, as it were, that are triggered by the same input. Many of the corresponding phenomena and achievement will likely be due to the complex interaction of a large class of instruments of our orchestra of mental capacities. In the present context, however, I am interested in the question whether there are subclasses of such phenomena that can be directly tied to structural and functional properties of a single subsystem, namely the Perceptual System. Therefore, I will make a tentative attempt to single out, among the huge class of phenomena that in some way or the other can be related to "multiperspectivity", a class of phenomena that appear to me as potential candidates for explanatory accounts in terms of specific design principles of the Perceptual System.

My claim is that the specific design features of the Perceptual System, as outlined above, yield phenomena of "multiperspecitivity" as a constitutive and 
functionally crucial consequence. I will refer to types of "multiperspectitivity" that are a direct consequence of properties of the Perceptual System as "intrinsic multiperspectivity". Accordingly, phenomena witnessing "intrinsic multiperspectivity" can be explained by intrinsic properties of the Perceptual System, without substantially referring to properties of higher-order interpretative systems, systems by which the phenomenal percept is generated, or to properties of interactions of higher-order systems. "Intrinsic multiperspectivity" is a theoretical (and thus, needless to say, an abstract and highly idealised) notion rather than a phenomenological one and refers to those internal structural and computational features of the Perceptual System by which it can, for a given sensory input, simultaneously provide at its interfaces to higher systems multiple "interpretations". These "interpretations" can be conflicting in the sense that the higher-order cognitive systems, by which meanings are assigned in terms of "external world" properties, assign mutually incoherent interpretations to them.

The notion of intrinsic multiperspectivity presupposes an internal structure that is sufficiently rich to yield the corresponding phenomena. If, as presently prevailing accounts of perception presuppose, the conceptual endowment of the Perceptual System were rather thin and were basically exhausted by sensory concepts, the class of phenomena pertaining to intrinsic multiperspectivity would be empty. Rather, all forms of "multiperspectivity" would be due to, say, inferential and interpretative properties of higher-order systems. If, however, empirical evidence speaks in favour of a rich and powerful conceptual structure of the Perceptual System, the property of intrinsic multiperspectivity emerges, as I will argue below, as a natural (and functionally important) consequence of the computational relation between sensory codes and conceptual forms.

Perceptual psychology abounds with phenomena that are eligible for providing examples of intrinsic multiperspectivity, notably those that are grouped under headings such as figure-ground segmentation, bi-stability, the proximal mode, amodal completion, vagueness, or ambiguity between entire mental "frames of reference". A well-known prototypical example is provided by phenomena that are grouped under the heading of a dual character of pictures. This notion basically refers to the phenomenon that pictures can generate an in-depth spatial impression of the scene depicted while at the same time appearing as flat two-dimensional surfaces hanging on a wall. In picture perception, we can simultaneously have the phenomenal impression of two different types of objects, each of which seems to thrive in its own autonomous spatial framework, namely, on the one hand, the picture surface as an object - with corresponding object properties such as orientation or depth - and, on the other hand, the depicted objects themselves with their idiosyncratic spatial properties and relations. We seem to have two mutually incompatible spatial representations at the same time; at least in the sense that they are available internally and we can, without any effort, switch between them. This aspect is phenomenally so conspicuous and striking that we usually do not pay much attention to it. Though such switches are correlated with depth aspects, they actually pertain to the entire perceptual organisation of the visual field and thus to attributes like shape, or shading and brightness gradients. 
Gombrich (1982) made the important observation that one has to achieve the proper "mental attitude" to take full advantage of the capacity to switch back and forth between the reality of the picture as an object and the reality of the depicted objects. Michotte (1948/1991) recognised the challenge that this kind of phenomenon poses for perception theory. Although a wealth of observations pertaining to this kind of phenomenon has accrued in the literature, our theoretical understanding continues to be poor [see Mausfeld (2003b), for a more detailed account].

Here, I will use the famous Heider-Simmel demonstration (cf. Scholl and Tremoulet 2000) to illustrate how intrinsic multiperspectivity is yielded as constitutive and functionally crucial consequence of the architectural and structural properties of the perceptual system outlined above. This demonstration seems to me particularly suited for making more conspicuous structural properties of our mental architecture that we otherwise find difficult to notice because they are an all pervading property of the way we are designed. In the Heider-Simmel demonstration, a small and a large triangle and a small circle move against and around each other and an open rectangle (see Fig. 7 for a movie still), and where observers unanimously perceive this event in terms of intentional concepts, such as chasing, looking for, hiding, conferring, being furious or frightened, etc. If, however, the movement of the figures is speeded up or slowed down these intentional impressions deteriorate or vanish entirely.

Obviously, the movement patterns of these geometrical objects suffices for eliciting a perceptual ascription of complex internal attributes pertaining to dispositional attributes of perceptual objects of the type "agent" or "mykind". Although in this demonstration, we undoubtedly and irresistibly perceive self-propelling objects with intentional attributes, we still are aware that we actually see only geometrical objects moving, again a kind of double book-keeping. As in the case of picture perception, one kind of percept does not vitiate the other, both can exist simultaneously as two layers of perceptual interpretation, as it were. Despite the fact that we ascribe in the Heider-Simmel demonstration anthropomorphic properties to the perceptual objects, it would never occur to us to actually interact with these objects.

Fig. 7 Movie still from the Heider and Simmel (1944) movie

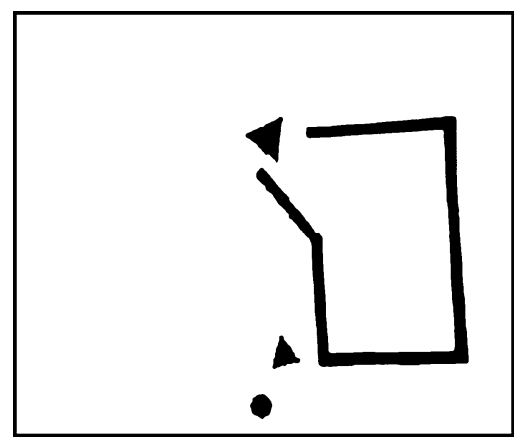


As the Heider-Simmel demonstration illustrates, our perceptual systems is highly sensitive to mechanical and intentional contingencies, which can activate perceptual "interpretations" that go far beyond any given sensory information. Via these contingencies, we segment our world into animate and inanimate objects and, in the case of non-mechanical contingencies or causation at a distance, attribute agency and internal intentions to a perceptual object. Perceptual objects of the type "animate object" can already be activated by appropriate temporal sequences of two-dimensional shapes (even pre-linguistic infants attribute intentionality to abstract shapes based solely upon spatio-temporal variables, e.g. Johnson 2003; Hamlin et al. 2007; Baillargeon et al. 2009). These perceptual achievements are part of our general capacity for making causal assignments and for embedding all of our experiences into various kinds of internal causal analyses. ${ }^{25}$ Due to this capacity we can visually attain aspects of "perceptual objects" that pertain to their "hidden" dispositional powers and propensities (which in the case of perceptual objects of the type "mykind" pertain to "mental states"). Empirical observations and theoretical considerations strongly suggest that the capability to mentally interact with others is part of the newborn's biological endowment, which quickly matures to a state where the child can impute mental states to oneself and to others. This biological endowment requires the availability of appropriate conceptual forms (whose nature is still at the boundary of scientific elucidation) that have their proprietary ways of exploiting the sensory input. The Heider-Simmel demonstration illustrates that the conceptual forms involved code properties that go far "beyond" those physico-geometrical properties that can be coded by sensory codes.

The notion of intrinsic multiperspectivity as employed here is tied to a specific theoretical conception of the functional architecture underlying perceptual achievements. While it is generally agreed that a perceptual system, however conceived, can in an idealised manner be distinguished from other systems of the mind and thus can be made an object of independent enquiry, ideas about how to conceive of this system, in particular assumptions about its conceptual and computational richness, diverge vastly. If it is assumed, as reigning theoretical frameworks in perceptual psychology do, that the conceptual apparatus of the perceptual system is confined to elementary sensory concepts, no issue of intrinsic multiperspectivity could arise. On such conceptions, as they also find their expression in the Standard Model of Perception, the entire explanatory burden with respect to phenomena such as the dual nature of pictures, the Heider-Simmel demonstration, amodal completion, etc. is placed upon higher-order interpretative systems. While this could have been possible in principle, corresponding conceptions are highly inadequate on both empirical and theoretical grounds, as pointed out above.

\footnotetext{
${ }^{25}$ All perceptions come, to use a seventeenth-century term, cum argumento causae. Accordingly, in the terminology employed here, the output of the Perceptual System is always couched in terms of internal causal analyses, whose specific forms are coded by conceptual forms of the type "event".
} 


\section{Intrinsic Multiperspectivity as the Activation of Multiple Layers of Underspecified Conceptual Forms}

The property of intrinsic multiperspectivity will emerge as a direct consequence of the type of functional architecture roughly indicated in Fig. 2. In contrast, this property will be well-nigh absent in simpler types of nervous systems in which the same type of sensory input can be independently exploited by different subsystems and simultaneously used for different tasks, but in which the integration of different subsystems is kept slim. Intrinsic multiperspectivity only shows up in more complex systems, in which an increasing number of modular subsystems with a rich computational machinery have been evolutionary interposed between the sensory input, on the one hand, and the behavioural effector systems, on the other hand. As a consequence of their qualification as a computational system, such systems have to be furnished with sufficiently abstract data types/conceptual forms over which their computational processes are defined. Due to the availability of abstract data types, these systems are prone to exhibit a pervasive and profound degree of inferential and conceptual integration. Because conceptual forms become increasingly decoupled from specific sensory inputs in corresponding architectures, it becomes almost unavoidable that each given sensory input yields a (partial) activation of a great variety of different types of conceptual forms. On the assumption that conceptual forms with a sufficient degree of activation are offered, at the corresponding interfaces, to higher-order systems, phenomena pertaining to intrinsic multiperspectivity are inescapable in these types of functional architecture.

While we can have some confidence, or so I believe, in the appropriateness of the general idea that the core principles of perception pertain to a triggering of conceptual forms, our understanding of the specific nature of these forms and of the associated computational mechanisms is still extremely thin. However, some more superficial aspects of the basic logic of an internal handling of underspecified conceptual forms can be illustrated by the Heider-Simmel phenomenon.

As is apparent from the phenomenal percept, the same sensory input, namely that provided by the Heider-Simmel movie, is exploited by two different types of conceptual forms: one pertaining to non-living physical objects and their attributes and the other pertaining to living objects of the type "mykind" (or "agent") and their internal attributes. Both types of conceptual forms are underspecified by this kind of sensory input. In particular, in conceptual forms of the type "mykind", intrinsic parameters for attributes pertaining to faces, eyes, limbs etc. cannot be specified by the given input. Rather, sensory codes pertaining to form aspects actually impede an activation of these types of conceptual forms. At the same time, sensory codes pertaining to motion patterns evidently foster an activation of the type "self-propelled object", "agent", or "mykind". Apparently, the Perceptual System attaches, with respect to the activation of these types of conceptual forms, more weight to the corresponding motion codes than to the form codes, in this situation. For conceptual forms of the type "non-biological physical objects", the triggering situation reverses. Here, the sensory codes for motion aspects vitiate an activation of these conceptual 
forms because the specific motion properties of the input violate internal constraints of the kinds of causal analyses associated with these types of conceptual forms (see Fig. 8 for an illustration of this triggering condition).

This broad description of the triggering situation is, first of all, a specific way of framing theoretical questions that arise in the context of the Heider-Simmel phenomenon. It is, needless to say, a far cry from being an explanation of it. An explanation would have to refer to independent descriptions of the nature of the conceptual forms involved and of the specific triggering function. Furthermore, it has to address, e.g. issues regarding the integration of sensory codes; the integration of the violations of various types of internal constraints; the computational means by which various fragments or aspects are brought together into a globally coherent pattern; and local and global evaluation functions. However, Fig. 7 makes clear that intrinsic multiperspectivity emerges as a natural feature within the assumed kind of functional architecture. The same input can simultaneously yield multiple conflicting "interpretations" at its interfaces to higher-order systems. In each of these conflicting "interpretations", part of the input or the sensory codes remains un-interpreted (either the self-motion of the objects and the kind of trajectories, or the form of the objects that carry intentional attributes). This is illustrated by Fig. 9 .

While the number of simultaneous "interpretations" provided by the Perceptual System is in principle only limited by the number of systematically connected packages of conceptual forms, computational requirements (pertaining, e.g. to stability aspects) and limitations of subsequent systems (in particular attentional ones) will reduce it to a very small value, usually down to two. Each of these "interpretations" embraces its own types of perceptual attributes; thrives in its own global framework; and is accompanied with its own degree of "realness".

The theoretical picture tentatively explored here helps to understand the important fact that (contrary to misconceptions that dominate reigning conceptions) "realness", or rather "unrealness", is a purely internal attribute that is assigned, on the basis of internal evaluation functions, to "interpretations" offered by the Perceptual System. The evaluation functions for assigning degrees of realness will presumably belong to higher levels at which phenomenal percepts are generated.

\section{SENSORY SYSTEM PERCEPTUAL SYSTEM}

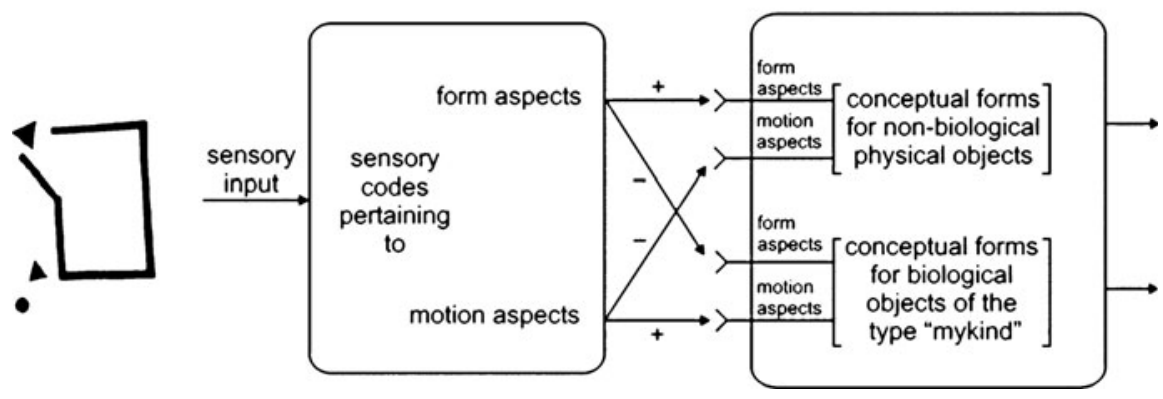

Fig. 8 Differential triggering of two different conceptual forms by the Heider-Simmel movie 
The triggering conditions for the assignment of the global attribute "real"/ "unreal" can be quite idiosyncratic with respect to our ordinary intuitions about what is real. Furthermore, the degree of perceptual salience of an "interpretation" of the Perceptual System and the degree of "realness" can be largely dissociated. In the Heider-Simmel case, the perception as "agents" with intentional attributes is more salient phenomenally; nevertheless, the "interpretation" in terms of moving geometric objects is assigned a higher degree of "realness".

Intrinsic multiperspectivity is not simply an odd by-product of the functional architecture of the perceptual system. It rather constitutes, for a system that is biologically endowed with a rich system of conceptual forms, an essential computationally element for dealing with internal problems pertaining to aspects of vagueness, ambiguity, and indeterminacy.

Its counterpart will most probably be found in all higher-order systems that take advantage of the output of the Perceptual System. Intrinsic multiperspectivity, in one way or the other, is a pervading property of our mental architecture. It is the

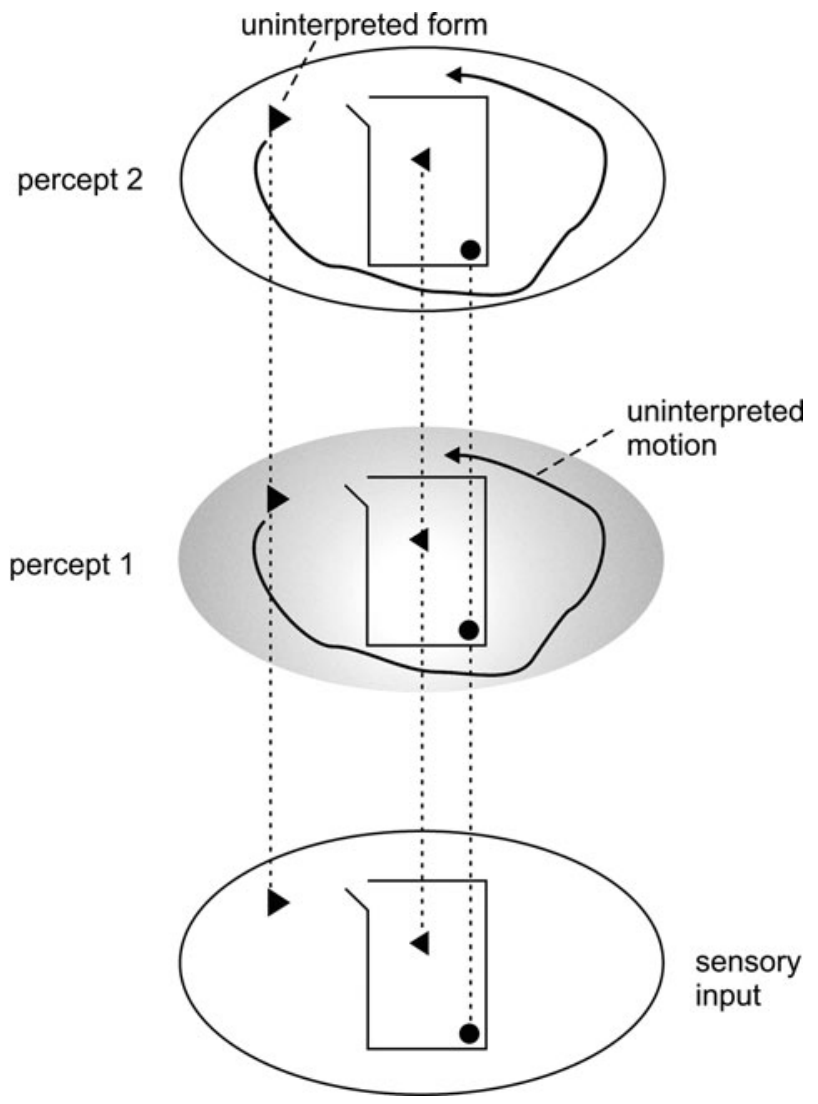

Fig. 9 Layers of conflicting perceptual "interpretation" triggered by the same sensory input 
foundation pillar of a stupendous range of capacities that allow us to simultaneously take conflicting perspectives in "looking at things and thinking about the products of our minds" (Chomsky 2000, p 36), capacities whose imprints range from simplest perceptual phenomena to our capability to read or to employ metaphors or allegories.

Acknowledgement This work was supported by BMBF-grant 01GWS060 and DFG-grants MA 1025/10-3 and 1025/10-4.

\section{References}

Ainsworth PM (2009) Newman's objection. Br J Philos Sci 60:135-171

Baillargeon R, Wu D, Yuan S, Li J, Luo Y (2009) Young infants' expectations about self-propelled objects. In: Hood B, Santos L (eds) The origins of object knowledge. Oxford University Press, Oxford, pp 285-352

Chomsky N (1975) Reflections on language. Pantheon Books, New York, NY

Chomsky N (1981) On the representation of form and function. Linguist Rev 1:3-40

Chomsky N (2000) New horizons in the study of language and mind. Cambridge University Press, Cambridge

Craton LG (1996) The development of perceptual completion abilities: infants' perception of stationary, partially occluded objects. Child Dev 67:890-904

Cudworth, R. (1731). A treatise concerning eternal and immutable morality. James and John Knapton, London: (Reprinted 1976 by Garland, New York)

Cottingham J (1986) Descartes. Blackwell, Oxford

Descartes R (1642/1985) Meditations of first philosophy. In: Cottingham J, Stoothoff R, Murdoch D (eds) The philosophical writings of Descartes, vol 2. Cambridge University, Cambridge, MA

Fodor JA (1980) Fixation of belief and concept acquisition. In: Piatelli-Palmarini M (ed) Language and learning: the debate between Jean Piaget and Noam Chomsky. Harvard University Press, Cambridge, MA, pp 142-149

Fodor (2003) Is it a bird? Problems with old and new approaches to the theory of concepts, Times Literary Supplement, January 17: 3-4

Gallistel CR (1998) Symbolic processes in the brain: the case of insect navigation. In: Scarborough $\mathrm{D}$, Sternberg S (eds) Methods, models and conceptual issues an invitation to cognitive science, vol 4. MIT, Cambridge, MA, pp 1-51

Gaukroger S (1990) The background to the problem of perceptual cognition. In: Gaukroger S (ed) Arnauld: on true and false ideas. Manchester University Press, Manchester

Goodwin B (2009) Beyond the Darwinian paradigm: understanding biological forms. In: Ruse M, Travis J (eds) Evolution: the first four billion years. Harvard University Press, Cambridge, MA, pp 299-312

Gombrich EH (1982) The image and the eye. Phaidon, Oxford

Grier M (2001) Kant's doctrin of transcedental illusion. Cambridge University Press, Cambridge

Hamlin JK, Wynn K, Bloom P (2007) Social evaluation by preverbal infants. Nature 450:557-559

Harland DP, Jackson RR (2000) 'Eight-legged cats' and how they see - a review of recent work on jumping spiders. Cimbebasia 16:231-240

Heider F, Simmel M (1944) An experimental study of apparent behaviour. Am J Psychol 57: 243-259

Helmholtz Hv (1855) Über das Sehen des Menschen. In: Vorträge und Reden. 4. Aufl., Bd.1, 1896. Vieweg, Braunschweig 
Jacobs DK, Nakanishi N, Yuan D, Camara A, Nichols SA, Hartenstein V (2007) Evolution of sensory structures in basal metazoa. Integr Comp Biol 47:712-723

James W (1890/1983) The principles of psychology. Harvard University Press, Cambridge, MA

Johnson SC (2003) Detecting agents. Philos Trans R Soc B Biol Sci 358:549-559

Kellman PJ, Spelke ES (1983) Perception of partly occluded objects in infancy. Cogn Psychol 15:483-524

Kirschner M, Gerhart J (1998) Evolvability. Proc Natl Acad Sci USA 95:8420-8427

Kitano H (2004) Biological robustness. Nat Rev Genet 5:826-837

Leslie AM (1987) Pretense and representation: the origins of 'theory of mind'. Psychol Rev 94:412-426

Ludlow P (2003) Referential semantics for I-languages? In: Antony LM, Hornstein N (eds) Chomsky and his critics. Blackwell, Oxford, pp 140-161

Mausfeld R (2002) The physicalistic trap in perception. In: Heyer D, Mausfeld R (eds) Perception and the physical world. Wiley, Chichester, pp 75-112

Mausfeld R (2003a) 'Colour' as part of the format of two different perceptual primitives: the dual coding of colour. In: Mausfeld R, Heyer D (eds) Colour perception: mind and the physical world. Oxford University Press, Oxford, pp 381-430

Mausfeld R (2003b) Competing representations and the mental capacity for conjoint perspectives. In: Hecht H, Schwartz B, Atherton M (eds) Inside pictures: an interdisciplinary approach to picture perception. MIT, Cambridge, MA, pp 17-60

Mausfeld R (2010a) Intrinsic multiperspectivity: on the architectural foundations of a distinctive mental capacity. In: Frensch PA, Schwarzer R (eds) Cognition and neuropsychology: international perspectives on psychological science, vol 1. Psychology, London, pp 95-116

Mausfeld R (2010b) The perception of material qualities and the internal semantics of the perceptual system. In: Albertazzi L, van Tonder G, Vishwanath D (eds) Perception beyond Inference. The information content of visual processes. MIT, Cambridge, MA, pp 159-200

Mausfeld R (2010c) Psychologie, Biologie, kognitive Neurowissenschaften. Zur gegenwärtigen Dominanz neuroreduktionistischer Positionen und zu ihren stillschweigenden Grundannahmen. Psychol Rundsch 61:180-190

Maxwell G (1970) Theories, perception, and structural realism. In: Colodny RG (ed) The nature and function of scientific theories. University of Pittsburgh Press, Pittsburgh, PA, pp 3-34

Michotte A (1948/1991) L'énigma psychologique de la perspective dans le dessin linéaire. Bulletin de la Classe des Lettres de l'Académie Royale de Belgique 34:268-288 (The psychological enigma of perspective in outline pictures. In: Thinès G, Costall A, Butterworth G (eds) (1991) Michotte's experimental phenomenology of perception, Erlbaum, Hillsdale, NJ)

Michotte A (1960/1991) Le réel et l'irréel dans l'image. Bulletin de la Classe des Lettres de l'Académie Royale de Belgique 46:330-344 (The real and the unreal in the image. In: Thinès G, Costall A, Butterworth G (eds) (1991), Michotte's experimental phenomenology of perception, Erlbaum, Hillsdale, NJ)

Miller GA (1979) A very personal history (occasional paper no. 1). Center for Cognitive Science, Massachusetts Institute of Technology, Cambridge, MA

Müller G (2007) Evo-devo: extending the evolutionary synthesis. Nat Rev Genet 8:943-949

Newman SA, Bhat R (2009) Dynamical patterning modules: a 'pattern language' for development and evolution of multicellular form. Int J Dev Biol 53:693-705

Nilsson DE, Gislén L, Coates MM, Skogh C, Garm A (2005) Advanced optics in a jellyfish eye. Nature 435:201-205

Pinkal M (1996) Radical underspecification. In: Dekker P, Stokhof M (eds) Proceedings of the 10th Amsterdam Colloquium, Institute for Logic, Language and Computation, ILLC Publications, Amsterdam, pp 587-606

Pustejovsky J (1998) The semantics of lexical underspecification. Folia Linguist 32:323-347

Roediger HL (2004) What happened to behaviorism, Presidential column, APS Observer

Russell B (1927) The analysis of matter. George Allen \& Unwin, London 
Scholl BJ, Tremoulet P (2000) Perceptual causality and animacy. Trends Cogn Sci 4:299-309

Spelke ES (2000) Core knowledge. Am Psychol 55:1233-1243

Spelke ES, Kinzler KD (2007) Core knowledge. Dev Sci 10(1):89-96

Spencer H (1855) The principles of psychology. Longman, Brown, Green and Longmans, London Tinbergen N (1951) The study of instinct. Clarendon, Oxford

Tse PU (1999) Volume completion. Cogn Psychol 39:37-68

von Szily A (1921) Stereoskopische Versuche mit Schattenrissen. Gräfes Archiv für Ophtalmologie 105:964-972

Wagner GP, Mezey J, Calabretta R (2005) Natural selection and the origin of modules. In: Callabaut W, Rasskin-Gutman D (eds) Modularity understanding the development and evolution of complex natural systems. MIT, Cambridge, MA, pp 33-49

Worrall J (1989) Structural realism: the best of both worlds? Dialectica 43:99-124

Yolton JW (1984) Perceptual acquaintance from Descartes to Reid. University of Minnesota Press, Minneapolis, MN

Yolton JW (1996) Perception and reality. A history from Descartes to Kant. Cornell University Press, Ithaca, NY 
黛 Springer

http://www.springer.com/978-3-642-11667-4

Interdisciplinary Anthropology

Continuing Evolution of Man

(Eds.)W. Welsch; W. Singer; A Wunder

2011, XII, 174 p., Hardcover

ISBN: $978-3-642-11667-4$ 